\title{
A liquid-state theory that remains successful in the critical region
}

\author{
D. Pini, G. Stell \\ Department of Chemistry, State University of New York \\ at Stony Brook, Stony Brook, New York 11794-3400, U.S.A. \\ N. B. Wilding \\ Department of Physics and Astronomy, University of Edinburgh \\ Edinburgh EH9 3JZ, U.K.
}

\begin{abstract}
A thermodynamically self-consistent Ornstein-Zernike approximation (SCOZA) is applied to a fluid of spherical particles with a pair potential given by a hard-core repulsion and a Yukawa attractive tail $w(r)=-\exp [-z(r-1)] / r$. This potential allows one to take advantage of the known analytical properties of the solution to the Ornstein-Zernike equation for the case in which the direct correlation function outside the repulsive core is given by a linear combination of two Yukawa tails and the radial distribution function $g(r)$ satisfies the exact core condition $g(r)=0$ for $r<1$. The predictions for the thermodynamics, the critical point, and the coexistence curve are compared here to other theories and to simulation results. In order to unambiguously assess the ability of the SCOZA to locate the critical point and the phase boundary of the system, a new set of simulations has also been performed. The method adopted combines Monte Carlo and finite-size scaling techniques and is especially adapted to deal with critical fluctuations and phase separation. It is found that the version of the SCOZA considered here provides very good overall thermodynamics and a remarkably accurate critical point and coexistence curve. For the interaction range considered here, given by $z=1.8$, the critical density and temperature predicted by the theory agree with the simulation results to about $0.6 \%$.
\end{abstract}

Prepared for the John Barker festschrift issue of Molecular Physics.

State University at Stony Brook College of Eng. and Appl. Sci. Report No. 754, Jan. 1998. 


\section{Introduction}

After applying their version of thermodynamic perturbation theory to square-well and Lennard-Jones fluids, John Barker and Doug Henderson characterized it as a "successful theory of liquids" [四]. And so it was. When tested against simulation results it proved to be impressively accurate at liquid-state densities and temperatures, unlike some versions of thermodynamic perturbation theory that had preceded it. And it bypassed the troubling lack of thermodynamic self-consistency associated with the direct use of the radial distribution functions obtained from the integral-equation theories then available, as well as yielding thermodynamic results as good or better than the best results obtainable from such integral equations.

These positive features became hallmarks of successful thermodynamic perturbation theories for simple fluids and were shared by the versions [2] that followed the Barker and Henderson work as well as an alternative perturbative approach set forth somewhat earlier by Hauge and Hemmer [3] that was based on using the inverse range of the attractive interaction rather than its strength as a perturbation parameter. Integral-equation approaches with improved self-consistency were also developed subsequently to yield accurate liquid-state thermodynamics [四].

Unfortunately, the accuracy of all these approaches begins to decrease substantially as one leaves the liquid-state region located slightly above the triple point in temperature and follows the liquid-gas coexistence curve in the density-temperature plane up to the critical region. In particular, the shape of the coexistence curve and location of the critical point are not accurately reproduced, nor are related critical parameters. In the case of the perturbation theories, it is not hard to understand why this is so. All of them are mean-field-like in nature, associated with coexistence curves that are quadratic close to the critical point, whereas the true coexistence curve is very nearly cubic. That is, in these theories one finds near the critical point a coexistence curve of the form

$$
T_{c}-T \approx A\left|\rho-\rho_{c}\right|^{x}, \quad x=2,
$$

where $\rho_{c}$ and $T_{c}$ are the critical values of number density $\rho$ and absolute temperature $T$, and $A$ is a constant. In contrast, in an exact treatment, one would expect to find $x$ close to 3 . In these theories the resulting $T_{c}$ is usually more than $5 \%$ too high and the critical compressibility factor $\left(P / \rho k_{B} T\right)_{c}$ is usually more than $10 \%$ too high. Here $P$ is the pressure, and $k_{B}$ is the Boltzmann constant.

The thermodynamics associated with the radial distribution function $g(r)$ obtained form various integral-equation approaches cannot be so neatly categorized. However, in the cases in which there are substantial discrepancies between the several paths available for obtaining thermodynamics from $g(r)$, the most reliable and accurate coexistence behavior is often obtained from evaluating the thermodynamics through the excess internal energy expressed in terms of an integral over the pair potential $w(r)$ weighted by $g(r)$. For continuum-fluid models the resulting critical behavior is typically mean-field like in the cases that we have studied, and thus subject to the same deficiencies as one approaches the critical region. In some integral-equation approaches that have been developed in order to insure a certain degree of thermodynamic consistency, the description of the critical 
region and of the phase diagram appears to be more problematic: for instance, the modified hypernetted chain (MHNC) theory [5] is indeed able to predict quite satisfactorily the liquid and the vapor branches of the coexistence curve of a simple fluid at low enough temperature, but it fails to converge close to the critical point, so that the two branches remain unconnected, and the position of the critical point is not given directly by the theory, but must be determined by extrapolation [6, 7]. The same kind of behavior [6, 8] is found also for the HMSA integral equation [the acronym coming from the fact that the theory 9] interpolates between the hypernetted chain (HNC) and the soft mean spherical approximation (SMSA)].

The self-consistent Ornstein-Zernike approximation (SCOZA) we consider here is not mean-field-like, and it remains highly accurate as one goes from liquid-state conditions to critical-point conditions. In particular the power $x$ in Eq. (1) was recently shown analytically to be given in the SCOZA by exactly 20/7 [10. And as we discuss in this paper, in the hard-core Yukawa fluid (HCYF) $T_{c}$ appears to be within $0.6 \%$ of its value as estimated by our simulation results. (Similarly, in recent three-dimensional lattice-gas studies [11, 12] the SCOZA $T_{c}$ was found to be within $0.2 \%$ of its estimated exact value). As described elsewhere [10, 12] the scaling behavior of the SCOZA thermodynamics is somewhat different from the simple scaling one expects to see in the exact thermodynamics, although those differences only begin to appear clearly when $\rho$ and $T$ are within less than $1 \%$ of their critical values. Closer to the critical point, the effective exponents defined above $T_{c}$ approach spherical-model values as the critical point is approached, whereas the exponents defined below $T_{c}$ do not. The exponents are discussed in Sec. 3.

The SCOZA was proposed some time ago by Høye and Stell [13, 14] but fast and accurate algorithms for evaluating its thermodynamic predictions were developed only recently 11, 12, 15. A sharp assessment of its accuracy for the HCYF could not be made on the basis of existing simulations, and for that reason our study here includes new Monte Carlo (MC) results exploiting finite-size scaling (FSS) techniques [16.

We have chosen the HCYF pair potential as the first of the continuum-fluid potentials to be considered in our studies of the SCOZA for several reasons. First, it embodies the two key features one requires in an off-lattice pair potential in order to consider both the liquid state and liquid-gas criticality - a highly repulsive core and an attractive well. Second, the HCYF proves to be particularly convenient to analyze using the SCOZA (the square-well fluid is far less convenient in this regard). Third, the functional form of the hard-core Yukawa potential makes it appropriate as a generic solvent-averaged interaction potential between polyelectrolytes and colloids as well as a generic simplefluid pair potential. For this reason it seems particularly useful to have an accurate theory for both the structure and thermodynamics of the HCYF, which has already been the subject of a number of previous studies. We shall make contact with several of those here.

The paper is organized as follows: in Sec. 2 we describe the theory and present some details of the method for the system under study, in Sec. 3 our results are shown and a comparison with other theories and simulation results is made, and in Sec. 4 our conclusions are drawn. The treatment of the hard-sphere gas and the main features of the MC-FSS simulation method are summarized respectively in Appendix A and Appendix B. 


\section{Theory}

Here we consider a fluid of spherical particles interacting via a two-body potential $v(r)$ which is the sum of a singular repulsive hard-sphere contribution and an attractive tail $w(r)<0$. The expression for $v(r)$ is then

$$
v(r)= \begin{cases}+\infty & r<1 \\ w(r) & r>1,\end{cases}
$$

where the hard-sphere diameter has been set equal equal to unity. As is customary in integral equation theories of fluids, the present approach introduces an approximate closure relation for the direct correlation function $c(r)$ which, once supplemented with the exact Ornstein-Zernike equation involving $c(r)$ and the radial distribution function $g(r)$, yields a closed theory for the thermodynamics and the correlations of the system under study. The basic requirement we want to incorporate in the SCOZA is the consistency between the compressibility and internal energy route to the thermodynamics. According to the compressibility route, the thermodynamics stems from the reduced compressibility $\chi_{\text {red }}$ as determined by the sum rule

$$
\chi_{\mathrm{red}}=\frac{1}{1-\rho \widehat{c}(k=0)},
$$

where $\widehat{c}(k)$ is the Fourier transform of the direct correlation function and $\rho$ is the number density of the system. In the internal energy route the key to the thermodynamics is instead provided by the excess internal energy as given by the integral of the interaction weighted by the radial distribution function:

$$
u=2 \pi \rho^{2} \int_{1}^{+\infty} d r r^{2} w(r) g(r),
$$

where $u$ is the excess internal energy per unit volume and we have taken into account that $g(r)$ vanishes for $r<1$ due to the hard-core repulsion. In the following we will refer

to the "excess internal energy" simply as the "internal energy". If $\chi_{\text {red }}$ and $u$ come from a unique Helmholtz free energy it is straightforward to find that one must have

$$
\frac{\partial}{\partial \beta}\left(\frac{1}{\chi_{\text {red }}}\right)=\rho \frac{\partial^{2} u}{\partial \rho^{2}},
$$

where $\beta=1 /\left(k_{B} T\right), T$ being the absolute temperature, and $k_{B}$ the Boltzmann constant. While this relation is of course satisfied by the exact compressibility and internal energy, this is not the case with those predicted by most integral equation theories. In order to cope with this lack of thermodynamic consistency, we consider the following closure to the Ornstein-Zernike equation:

$$
\begin{cases}g(r)=0 & r<1, \\ c(r)=c_{\mathrm{HS}}(r)+K(\rho, \beta) w(r) & r>1,\end{cases}
$$


where $c_{\mathrm{HS}}(r)$ is the direct correlation function of the hard-sphere fluid, and $K(\rho, \beta)$ is a function of the thermodynamic state of the system. In Eq. (6) the approximation clearly lies in the simple form of $c(r)$ outside the repulsive core. The closure above resembles the one used in the approximation known as both the lowest-order gamma-ordered approximation (LOGA) [17] and the optimized random phase approximation (ORPA) 18]. However, while in the LOGA/ORPA one has $K(\rho, \beta) \equiv-\beta$, in Eq. (6) $K(\rho, \beta)$ is not fixed a priori, but instead must be determined so that the thermodynamic consistency condition (5) is satisfied. This gives rise to a partial differential equation (PDE) for the function $K(\rho, \beta)$, provided an expression for the hard-sphere direct correlation function $c_{\mathrm{HS}}(r)$ is given. The most popular parameterization for $c_{\mathrm{HS}}(r)$ in the fluid region is due to Verlet and Weis [19]. Another choice that yields the same thermodynamics as VerletWeis, and that we find convenient in view of the calculations performed in this work, is originally due to Waisman [20]. It was subsequently extended analytically by Høye and Stell [21] and explored in some detail by Henderson and coworkers [22]. It amounts to assuming that the function $c_{\mathrm{HS}}(r)$ outside the repulsive core has a one-Yukawa form, so that for the hard-sphere system we have:

$$
\begin{cases}g_{\mathrm{HS}}(r)=0 & r<1 \\ c_{\mathrm{HS}}(r)=K_{1} \frac{\exp \left[-z_{1}(r-1)\right]}{r} & r>1\end{cases}
$$

The Ornstein-Zernike equation supplemented by Eq. (7) can be solved analytically in terms of the amplitude $K_{1}$ and the inverse range $z_{1}$ of $c_{\mathrm{HS}}(r)$. These can be in turn determined as a function of the density by requiring, as in the Verlet-Weis parameterization, that both the compressibility and the virial route to the thermodynamics give the Carnahan-Starling equation of state. The basic features of the calculation are recalled in Appendix A.

A considerable, although purely technical, simplification in the closure scheme outlined above based on Eqs. (5), (6) occurs when also the attractive potential $w(r)$ in Eq. (2) is given by a Yukawa function, i.e. when one has

$$
w(r)=-\frac{\exp [-z(r-1)]}{r}
$$

$z$ being the inverse range of the potential. From Eq. (17) it is then immediately seen that Eq. (6) becomes

$$
\begin{cases}g(r)=0 & r<1, \\ c(r)=K_{1} \frac{\exp \left[-z_{1}(r-1)\right]}{r}+K_{2} \frac{\exp \left[-z_{2}(r-1)\right]}{r} & r>1,\end{cases}
$$

where $K_{2}$ and $z_{2}$ are the quantities referred to as $K$ and $z$ in Eq. (ब), (\$), and $K_{1}, z_{1}$ are known function of the density. It is now possible to take advantage of the fact that for the Ornstein-Zernike equation supplemented by the closure (9) extensive analytical results 
have been determined [23, 24, 14]. If both $K_{1}$ and $K_{2}$ are given, as in the LOGA/ORPA, this enables one to solve Eq. (9) altogether [25, 26, 27]. More generally, irrespective of the form of $K_{1}$ and $K_{2}$, a prescription can be found to determine the reduced compressibility $\chi_{\text {red }}$ as a function of the density $\rho$ and the internal energy per unit volume $u$, which can be used in Eq. (5) to obtain a closed PDE. A similar procedure for the same potential considered here was adopted in a previous work [15], where however the hard-sphere contribution to the direct correlation function $c_{\mathrm{HS}}(r)$ outside the core was not taken into account, so that $c(r)$ was given by a simple one-Yukawa tail. This further simplifies the theory, but implies that the description of the hard-sphere fluid coincides with that of the PY approximation, which as is well known is not very satisfactory at high density. This defect becomes more and more severe as the range of the attractive interaction decreases, and can considerably affect the phase diagram predicted by the theory, unless some moreor-less ad hoc procedure is adopted to correct the hard-sphere thermodynamics. In order to incorporate a better treatment of the hard-sphere fluid into the theory one can turn to the two-Yukawa form for $c(r)$ of Eq. (9), whose use in the consistency condition (5) we are now going to illustrate in some detail. In the following we will exploit the results determined in Refs. 223, 24, 14, which will be respectively referred to as I, II, III. Let us introduce the packing fraction $\xi=\pi \rho / 6$ and the quantity

$$
f=(1-\xi) \sqrt{\frac{1}{\chi_{\text {red }}}},
$$

which is the square root of the quantity referred to as $A$ in I, II, III. Eq. (5) becomes

$$
\frac{2 f}{(1-\xi)^{2}}\left(\frac{\partial f}{\partial u}\right)_{\rho}\left(\frac{\partial u}{\partial \beta}\right)_{\rho}=\rho\left(\frac{\partial^{2} u}{\partial \rho^{2}}\right)_{\beta} .
$$

To obtain a PDE for $u$ we need to express $f$ as a function of $\rho$ and $u$ in Eq. (11). From Eq. (II.14) it is found that $f$ can be written as

$$
f=-\frac{\left(z_{1}^{2}-z_{2}^{2}\right)+4 \sqrt{q}\left(\gamma_{2}-\gamma_{1}\right)}{4\left[\left(z_{1} / z_{2}\right) \gamma_{2}-\left(z_{2} / z_{1}\right) \gamma_{1}\right]}-\frac{z_{1}^{2}-z_{2}^{2}}{z_{1} z_{2}} \frac{\gamma_{1} \gamma_{2}\left(\gamma_{2}-\gamma_{1}\right)}{\left[\left(z_{1} / z_{2}\right) \gamma_{2}-\left(z_{2} / z_{1}\right) \gamma_{1}\right]^{2}}
$$

where we have set

$$
q=\frac{(1+2 \xi)^{2}}{(1-\xi)^{2}}
$$

The quantities $\gamma_{1}$ and $\gamma_{2}$ are given by Eq. (II.5)

$$
\begin{aligned}
& \gamma_{1}=2-\sqrt{q}-\frac{U_{1}}{U_{0}}, \\
& \gamma_{2}=2-\sqrt{q}-\frac{W_{1}}{W_{0}} .
\end{aligned}
$$

The ratios $U_{1} / U_{0}$ and $W_{1} / W_{0}$ depend on the integrals

$$
I_{i}=4 \pi \rho \int_{1}^{+\infty} d r r \exp \left[-z_{i}(r-1)\right] g(r) \quad(i=1,2) .
$$


¿From Eq. (I.35) it is found in fact

$$
\frac{W_{1}}{W_{0}}=\frac{4+2 z_{2}-z_{2}^{2}}{2\left(2+z_{2}\right)} \frac{\tau_{2} I_{2}-1}{\sigma_{2} I_{2}-1}
$$

and the corresponding relation with $W_{1} / W_{0}$ replaced by $U_{1} / U_{0}$ and the index 2 changed to 1 . The quantities $\tau_{i}$ and $\sigma_{i}$ depend only on $z_{i}$ and are given by Eq. (I.34):

$$
\begin{aligned}
\sigma_{i} & =\frac{1}{2 z_{i}}\left[\frac{z_{i}-2}{z_{i}+2}+\exp \left(-z_{i}\right)\right], \\
\tau_{i} & =\frac{1}{2 z_{i}}\left[\frac{z_{i}^{2}+2 z_{i}-4}{4+2 z_{i}-z_{i}^{2}}+\exp \left(-z_{i}\right)\right],
\end{aligned}
$$

with $i=1,2$. From the expression of the potential (8) it is readily seen that $I_{2}$ is directly related to the internal energy per unit volume $u$ given by Eq. (4):

$$
u=-\frac{1}{2} \rho I_{2}
$$

Eqs. (15), (17), (20) allow then to express $\gamma_{2}$ explicitly as a function of $\rho$ and $u$ :

$$
\gamma_{2}=2-\sqrt{q}-\frac{4+2 z_{2}-z_{2}^{2}}{2\left(2+z_{2}\right)} \frac{2 \tau_{2} u+\rho}{2 \sigma_{2} u+\rho} .
$$

We now need $\gamma_{1}$ as a function of $\rho$ and $u$. This is less straightforward than for $\gamma_{2}$, since the integral $I_{1}$ does not have any direct thermodynamic meaning, the exponential in $I_{1}$ being related to the tail of the direct correlation function of the hard-sphere gas. We have then to make use of some further results determined in I-III. ¿From Eq. (I.36) it is found that the amplitudes $K_{1}, K_{2}$ of the Yukawa functions in the closure (9) can be expressed in terms of the above introduced quantities $U_{0}, U_{1}, W_{0}, W_{1}$. One has

$$
K_{1}=\frac{2\left(z_{1}+2\right)^{2} \sigma_{1}^{2}}{3 \xi z_{1}^{2}} U_{0}\left[\frac{U_{1}}{U_{0}}-\alpha_{1}\right]^{2}
$$

where $\alpha_{1}$ is given by Eq. (I.37):

$$
\alpha_{1}=\frac{\left(4+2 z_{1}-z_{1}^{2}\right) \tau_{1}}{2\left(2+z_{1}\right) \sigma_{1}},
$$

and the corresponding equations with the index 1 replaced by 2 and $U_{0}, U_{1}$ replaced by $W_{0}, W_{1}$. Let us now introduce the quantities $x, y$ given by

$$
\begin{aligned}
& x=\sqrt{q}-\frac{z_{1}^{2}}{4 \gamma_{1}}, \\
& y=\sqrt{q}-\frac{z_{2}^{2}}{4 \gamma_{2}} .
\end{aligned}
$$


¿From Eq. (III.30) one has

$$
\begin{aligned}
U_{0} & =\frac{4}{z_{1}^{2}} p(\sqrt{q}-x)^{2}, \\
W_{0} & =\frac{4}{z_{2}^{2}} s(\sqrt{q}-y)^{2} .
\end{aligned}
$$

where $p$ and $s$ must satisfy Eq. (II.39) (in the notation of II one has $x \equiv u_{q 1} / u_{q 0}, y \equiv$ $\left.w_{q 1} / w_{q 0}, p \equiv u_{q 0}, s \equiv w_{q 0}\right)$ :

$$
\left\{\begin{array}{l}
p+s+\frac{4 s}{z_{1}^{2}-z_{2}^{2}}(y-x)^{2}=\frac{1}{4} z_{1}^{2}-x^{2}, \\
p+s-\frac{4 p}{z_{1}^{2}-z_{2}^{2}}(y-x)^{2}=\frac{1}{4} z_{2}^{2}-y^{2} .
\end{array}\right.
$$

Eq. (28) is readily solved for $p$ and $s$ to give

$$
p=-\frac{z_{1}^{2}-z_{2}^{2}}{64(y-x)^{4}}\left\{4 z_{2}^{2}(y-x)^{2}-16 y^{2}(y-x)^{2}-\left(z_{1}^{2}-z_{2}^{2}\right)\left[z_{1}^{2}-z_{2}^{2}+4\left(y^{2}-x^{2}\right)\right]\right\},
$$

and the expression for $s$ is obtained by exchanging $z_{1}, z_{2}$ and $x, y$ in the r.h.s. of Eq. (29). If Eqs. (14), (24), (26), (29) are used in Eq. (22) we finally obtain

$$
\begin{aligned}
& {\left[4\left(2-\sqrt{q}-\alpha_{1}\right)(\sqrt{q}-x)-z_{1}^{2}\right]^{2}\left\{4 z_{2}^{2}(y-x)^{2}-16 y^{2}(y-x)^{2}\right.} \\
& \left.\quad-\left(z_{1}^{2}-z_{2}^{2}\right)\left[z_{1}^{2}-z_{2}^{2}+4\left(y^{2}-x^{2}\right)\right]\right\}=-\frac{384 \xi z_{1}^{4}}{\left(z_{1}+2\right)^{2}\left(z_{1}^{2}-z_{2}^{2}\right) \sigma_{1}^{2}} K_{1}(y-x)^{4},
\end{aligned}
$$

and a similar equation obtained by exchanging the indices 1 and 2 and the quantities $x, y$. We recall that in Eq. (30) $K_{1}, z_{1}, \sigma_{1}$, and $\alpha_{1}$ are known functions of the density $\rho$ which refer to the hard-sphere system. For given values of $\rho$ and $u$, Eqs. (21), (25) allow one to determine $y$. Eq. (30) can then be solved numerically with respect to $x$ to obtain $\gamma_{1}$ via Eq. (24). This solves the problem of determining $\gamma_{1}$ in terms of $\rho$ and $u$. The partial derivative $(\partial f / \partial u)_{\rho}$ that appears in Eq. (11) can then be determined as

$$
\left(\frac{\partial f}{\partial u}\right)_{\rho}=\left(\frac{\partial f}{\partial \gamma_{1}}\right)_{\rho}\left(\frac{\partial \gamma_{1}}{\partial u}\right)_{\rho}+\left(\frac{\partial f}{\partial \gamma_{2}}\right)_{\rho}\left(\frac{\partial \gamma_{2}}{\partial u}\right)_{\rho},
$$

where $\left(\partial \gamma_{2} / \partial u\right)_{\rho}$ is calculated explicitly by Eq. (21), while $\left(\partial \gamma_{1} / \partial u\right)_{\rho}$ must be determined as the derivative of the function implicitly defined by Eq. (30). If we write Eq. (30) as $F(x, y, \rho)=0$, it is found straightforwardly that Eq. (11) takes the form

$$
B(\rho, u) \frac{\partial u}{\partial \beta}=C(\rho, u) \frac{\partial^{2} u}{\partial \rho^{2}},
$$

where the functions $B(\rho, u)$ and $C(\rho, u)$ are given by the following expressions:

$$
\begin{aligned}
B(\rho, u) & =\frac{2 f}{(1-\xi)^{2}} \frac{\partial \gamma_{2}}{\partial u}\left[\frac{\partial f}{\partial \gamma_{2}} \frac{\partial F}{\partial x} \frac{\partial x}{\partial \gamma_{1}}-\frac{\partial f}{\partial \gamma_{1}} \frac{\partial F}{\partial y} \frac{\partial y}{\partial \gamma_{2}}\right], \\
C(\rho, u) & =\rho \frac{\partial F}{\partial x} \frac{\partial x}{\partial \gamma_{1}} .
\end{aligned}
$$


All the partial derivatives in Eqs. (33), (34) are calculated at constant $\rho$ and can be determined by Eqs. (12), (21), (24), (25), (30). The resulting expressions are then evaluated as a function of $\rho$ and $u$ via the procedure described above. The same procedure also allows one to determine the reduced compressibility as $1 / \chi_{\text {red }}=f^{2} /(1-\xi)^{2}$ once $f$ has been obtained from Eq. (12). The PDE (32) is a non-linear diffusion equation that must be integrated numerically. To prevent the occurrence of any numerical instability, especially in the critical and sub-critical region, we have adopted an implicit finite-differences algorithm [28] tailored to equations that, although globally non-linear, depend on the partial derivatives of the unknown function in a linear fashion like Eq. (32). The integration with respect to $\beta$ starts at $\beta=0$ and goes down to lower and lower temperatures. Before each integration step Eq. (30) is solved numerically and the coefficients $B(\rho, u), C(\rho, u)$ are determined. The density $\rho$ ranges in a finite interval $\left(0, \rho_{0}\right)$, whose high-density boundary has been typically set at $\rho_{0}=1$. The initial condition can be determined by taking into account that at $\beta=0$ the radial distribution function coincides with that of the hard-sphere gas. From Eqs. (4) and (8) one has then

$$
u(\rho, \beta=0)=-2 \pi \rho^{2} \int_{0}^{+\infty} d r r \exp \left[-z_{2}(r-1)\right] g_{\mathrm{HS}}(r) \quad \text { for every } \rho,
$$

where $g_{\mathrm{HS}}(r)$ is obtained in the present scheme by the closure (7). For such a closure, as shown in Appendix A, both $U_{0}$ and $U_{1}$ in Eq. (14) can be determined analytically as a function of $\rho$, thus providing $\gamma_{1}(\rho)$ at $\beta=0$. This allows one to obtain $u$ in Eq. (35) analytically as well: in fact, one can solve Eq. (12) for $\gamma_{2}$ as a function of $\gamma_{1}, f$, and $\rho$, where $f$ is readily obtained by using the Carnahan-Starling expression of $\chi_{\text {red }}$ in Eq. (10). Once $\gamma_{2}$ is known, Eq. (21) is solved with respect to $u$. It must be noted that solving Eq. (12) for $\gamma_{2}$ gives two branches, so attention must be paid in order to single out the branch that actually corresponds to the physical solution. We also need two boundary conditions at $\rho=0$ and $\rho=\rho_{0}$. ¿From Eq. (4) one has immediately

$$
u(\rho=0, \beta)=0 \quad \text { for every } \beta .
$$

At high density we instead make use of the so-called high-temperature approximation (HTA), according to which the excess Helmholtz free energy per unit volume is determined via Eq. (35) for every temperature. In the fluid region of the phase diagram this of course is not exact unless $\beta=0$, but it becomes more and more accurate as the density of the system is increased [29], so we expect that for a given sweep along the $\beta$-axis the results will not differ appreciably from what would be obtained using an hypothetical exact boundary condition, provided the boundary $\rho_{0}$ is located at sufficiently high density. We used the HTA at $\rho=\rho_{0}$ for the reduced compressibility. This yields via Eq. (5) the boundary condition

$$
\frac{\partial^{2} u}{\partial \rho^{2}}\left(\rho_{0}, \beta\right)=\frac{\partial^{2} u}{\partial \rho^{2}}\left(\rho_{0}, \beta=0\right) \quad \text { for every } \beta .
$$

We have checked that the output of the numerical integration of Eq. (32) is quite insensitive to the specific choice of the high-density boundary condition. Moreover, for 
$\rho_{0} \simeq 1$ moving the boundary condition to higher densities also leaves the results unaffected. Eq. (10) shows that to be physically meaningful, the quantity $f$ has to be non-negative. On the other hand, below the critical temperature the solution of Eq. (32) does not satisfy this condition along the whole density interval $\left(0, \rho_{0}\right)$, but only outside a certain temperature-dependent region $\left(\rho_{s 1}(\beta), \rho_{s 2}(\beta)\right)$. For $\rho=\rho_{s 1}(\beta)$ or $\rho=\rho_{s 2}(\beta)$ the quantity $f$ vanishes, and consequently the compressibility diverges. As $\beta$ changes, $\rho_{s 1}(\beta)$ and $\rho_{s 2}(\beta)$ give then respectively the low- and the high- density branch of the spinodal curve predicted by the theory. The fact that $f$ becomes negative for $\rho_{s 1}(\beta)<\rho<\rho_{s 2}(\beta)$ not only implies that the theory behaves unphysically in this interval, but it also gives rise to an analytical instability which would make the numerical integration of the PDE (32) impossible, if one tried to determine the solution over the whole interval $\left(0, \rho_{0}\right)$ even below the critical temperature. Therefore, the region bounded by the spinodal has been excluded from the integration of Eq. (32). Specifically, as soon as it is found that $f$ changes sign, so that for a certain density $\widetilde{\rho}$ one has $f(\widetilde{\rho}, \beta)<0$, the integration is restricted to the interval $(0, \widetilde{\rho}-\Delta \rho)$ or $\left(\widetilde{\rho}+\Delta \rho, \rho_{0}\right)$ respectively for $\widetilde{\rho}<\rho_{c}$ or $\widetilde{\rho}>\rho_{c}$, where $\Delta \rho$ is the spacing of the density grid. Within the precision of the numerical discretization, one has $\rho_{s 1}=\widetilde{\rho}-\Delta \rho\left(\right.$ or $\left.\rho_{s 2}=\widetilde{\rho}+\Delta \rho\right)$ and the further boundary conditions

$$
u\left(\rho_{s i}, \beta\right)=u_{S}\left(\rho_{s i}\right) \quad i=1,2, \beta>\beta_{c},
$$

where $\beta_{c}$ is the critical inverse temperature and $u_{S}(\rho)$ is the value of the internal energy per unit volume when the compressibility at density $\rho$ diverges. This can be determined by setting $f=0$ in Eq. (12) and solving for $\gamma_{1}$ as a function of $\rho$ and $\gamma_{2}$. If Eqs. (24) and (25) are substituted into Eq. (30), an equation for $\gamma_{2}$ is obtained that allows one to determine the value of $\gamma_{2}$ when $1 / \chi_{\text {red }}=0$ for a certain $\rho$. Solving Eq. (21) with respect to $u$ then yields $u_{S}(\rho)$.

Once the internal energy per unit volume $u$ has been determined from Eq. (32), the pressure $P$ and the chemical potential $\mu$ are obtained by integration with respect to $\beta$ via the relations $\partial(\beta P) / \partial \beta=-u+\rho \partial u / \partial \rho, \partial(\beta \mu) / \partial \beta=\partial u / \partial \rho$. Thanks to the selfconsistency of the theory, this route to the thermodynamics is equivalent to integrating the inverse compressibility with respect to $\rho$, but it does not require one to circumvent the forbidden region in order to reach the high-density branch of the subcritical isotherms.

\section{Results}

The numerical integration of the PDE (32) with the initial condition (35) and the boundary conditions (36)-(38) has been performed on a density grid with $\Delta \rho=10^{-3}-10^{-4}$. At the beginning of the integration the temperature step $\Delta \beta$ was usually set at $\Delta \beta=$ $2 \times 10^{-5}-10^{-5}$. As the temperature approaches its critical value, $\Delta \beta$ can be further decreased if one wishes to get very close to the critical point, and then gradually expanded back. The integration was usually carried down to $\beta \simeq 2.4 \beta_{c}$. The inverse range parameter of the attractive tail in Eq. (8) has been set at $z=1.8$. For this value of $z$ several simulations [30, 31, 32] and theoretical [6, 26, 27] predictions have already been reported in the literature. Fig. 1 shows the SCOZA results for the compressibility factor 
$Z=P /\left(\rho k_{B} T\right)$ along two different isotherms, corresponding to $T=2$ and $T=1.5$, together with the $\mathrm{MC}$ simulation results by Henderson and coworkers [30]. The agreement is very good both at low and high density. The compressibility factors are also reported in Tab. 1, together with those obtained by the LOGA/ORPA via the internal energy route [27], which is the one that gives the best agreement with the simulation results. It can be seen that for the non-critical states reported here the SCOZA and the energy route of the LOGA/ORPA are very close to each other. In Tab. 2 the predictions for the chemical potential and the reduced compressibility are compared to the data from the MC simulations performed in this work. The internal energy per particle is reported in Tab. 3, where again the LOGA/ORPA results are also shown. The critical point predicted by the theory has been located by the vanishing of the inverse compressibility $1 / \chi_{\text {red }}$. No extrapolation procedure to $1 / \chi_{\text {red }}=0$ is necessary, since the algorithm adopted here allows one to get as close as desired to the critical singularity. As mentioned in Sec. 2, below the critical temperature $T_{c}$ the theory yields a spinodal curve. The coexistence curve must be determined by a Maxwell construction, i.e. by imposing the equilibrium conditions $\mu\left(\rho_{g}, T\right)=\mu\left(\rho_{l}, T\right), P\left(\rho_{g}, T\right)=P\left(\rho_{l}, T\right)$ for the densities $\rho_{g}, \rho_{l}$ of the gas and liquid phases at coexistence at a temperature $T$. In comparing our results for the critical point and the coexistence curve with the available simulation data, we found that the two simulations for the phase diagram of the system under study already reported in the literature [31, 32] do not agree very well with each other. We then performed a new set of simulations using the MC-FSS method summarized in Appendix B. The SCOZA and the simulation results for the critical point are compared in Tab. 4, which also shows the predictions of other theories [6]. It can be seen that the agreement between the SCOZA and the present simulation is remarkably good: the error in the critical density and temperature is respectively slightly more and slightly less than $0.6 \%$. The SCOZA and the simulation coexistence curve in the temperature-density plane are compared in Fig. 2. A similar comparison in the temperature-internal energy and in the temperature-chemical potential plane is shown respectively in Fig. 3 and in Fig 4 . In every case the SCOZA agrees very well with the simulation. It can be also appreciated that in the SCOZA the coexistence curve extends up to the critical point, while, as already observed in the Introduction, this is not always the case with other theories. In Tab. 4 and in Figs. 2-4 we have also reported the predictions of the simpler version of the SCOZA mentioned in Sec. 2, in which the direct correlation function outside the repulsive core is given by just one Yukawa tail, and the thermodynamics of the hard-sphere gas is described in the PY approximation. It can be observed that even for not very short-ranged interaction the treatment of the repulsive contribution considerably affects the phase diagram predicted by the theory, the two-Yukawa SCOZA sensibly improving over the one-Yukawa version. The behavior of the SCOZA in the critical region has been studied both analytically [10] and numerically [12]. This investigation has shown that above the critical temperature the SCOZA yields the same critical exponents as the mean spherical approximation (MSA), i.e. $\gamma=2, \delta=5, \alpha=-1$, where the usual notation for the critical exponents has been used. On the other hand, on the coexistence curve the critical exponents are neither spherical nor classical, and one finds $\gamma^{\prime}=7 / 5, \alpha^{\prime}=-1 / 10, \beta=7 / 20$ (here of course $\beta$ is the critical exponent that gives the curvature of the coexistence curve near the critical 
point). Although these results were determined in the case of a nearest-neighbor attractive lattice gas, we expect them to hold also in the continuum case. Fig. 5 shows the reduced compressibility of the HCYF for $T>T_{c}$ and $\rho=\rho_{c}$ as a function of the reduced temperature $t=\left(T-T_{c}\right) / T_{c}$ on a log-log plot. Also shown is the correspondent effective exponent $\gamma_{\mathrm{eff}}$, defined as the local slope of the plot. It can be seen that $\gamma_{\mathrm{eff}}$ eventually saturates at $\gamma=2$, thus signaling the onset of a MSA-like power-law behavior, but the asymptotic regime can be detected only at very small reduced temperature $\left(t \sim 10^{-6}\right)$. This is the same scenario previously found in the nearest-neighbor lattice gas. For the $\mathrm{HCYF}$, the crossover is controlled as expected by the inverse range parameter $z$. It has been verified that as the interaction becomes longer and longer ranged, the asymptotic regime is further pushed to smaller and smaller values of the reduced temperature $t$ [33].

\section{Conclusions}

We have studied the thermodynamics and the phase diagram of the HCYF using both the SCOZA and MC simulations supplemented by a finite-size scaling analysis. The comparison between theory and simulation results shows that the SCOZA yields both very good overall thermodynamics and a remarkably accurate coexistence curve up to the critical point. The version of the SCOZA considered here takes into account the hard-sphere contribution to the direct correlation function outside the repulsive core, and sensibly improves over the simpler one-Yukawa version, in which the hard-sphere gas is described as in the PY approximation. On the other hand, as stated in Sec. 2, here (as well as in the simpler version just mentioned) consistency has been enforced between the internal energy and the compressibility route, but not between the virial route and either of the above. We think that the further development of making the theory fully self-consistent by taking also the virial route into account is worth pursuing, since we anticipate that the present version of the SCOZA will yield liquid-state pressures from the virial theorem that are not significantly better than those obtained using the virial theorem with the LOGA/ORPA $g(r)$. We defer a detailed examination of this issue to a later study. In this respect it is worth mentioning an investigation of the HCYF along the lines considered here [34], where some results for the critical parameters were reported taking into account all the three routes to the thermodynamics although, as explicitly pointed out by the authors, the SCOZA equations were studied in an approximate fashion, and no attempt to determine the phase diagram was made.

Although dealing with a Yukawa potential entails certain analytical simplifications in implementing the SCOZA, such an approach can be applied to any kind of tail potential. It should also be pointed out that the idea of using the requirement of self-consistency to get a closed theory of thermodynamics and correlations is pertinent not only to the realm of simple fluids or lattice gases, but has also proven to be a powerful tool in the study of a system of spins with continuous symmetry [35], and of a site-diluted [36] or random-field [37] Ising model. 
D.P. gratefully acknowledges the support of the Division of Chemical Sciences, Office of Basic Energy Sciences, Office of Energy Research, U.S. Department of Energy. G.S. gratefully acknowledges the support of the National Science Foundation. N.B.W. acknowledges support from the EPSRC (grant number GR/L91412), and the Royal Society of Edinburgh.

\section{A Waisman parameterization of $c_{\mathrm{HS}}(r)$}

In this Appendix we recall the procedure that allows one to determine analytically the amplitude $K_{1}$ and the inverse range $z_{1}$ of the direct correlation function $c_{\mathrm{HS}}(r)$ of the hardsphere gas in the Waisman parameterization (7). The relevant equations are reported in Ref. [21], which will be referred here as IV. Both $z_{1}$ and $K_{1}$ are conveniently expressed in terms of two quantities $V_{0}, V_{1}$ which are formally analogous to $U_{0}, U_{1}$ and $W_{0}, W_{1}$ introduced in Eqs. (14), (15). ¿From Eq. (IV.2.32 a) it is found that $z_{1}$ is given by

$$
z_{1}=\frac{2}{q-f^{2}}\left[\left(V_{0}+f^{2}-q\right) f+\sqrt{\left(V_{0}+f^{2}-q\right) V_{0} q}\right],
$$

where $f$ and $q$ are defined in Eqs. (10), (13). The expression of the amplitude $K_{1}$ is the same as in Eqs. (22) with $U_{0}, U_{1}$ replaced by $V_{0}, V_{1}$ :

$$
K_{1}=\frac{2\left(z_{1}+2\right)^{2} \sigma_{1}^{2}}{3 \xi z_{1}^{2}} V_{0}\left[\frac{V_{1}}{V_{0}}-\alpha_{1}\right]^{2},
$$

where $\xi$ is the packing fraction $\xi=\pi \rho / 6$ and $\alpha_{1}$ is a function of $z_{1}$ given by Eq. (23). The ratio $V_{1} / V_{0}$ can be expressed as a function of $V_{0}$ by Eqs. (IV.2.24) and (IV.2.26). One has

$$
\frac{V_{1}}{V_{0}}=2-\sqrt{q}-\frac{1}{2 V_{0} \sqrt{q}}\left[\left(V_{0}+f^{2}-q\right)\left(V_{0}+f^{2}\right)+\frac{1}{4} z_{1}^{2}\left(q-f^{2}\right)\right] .
$$

To obtain the explicit expressions of $z_{1}$ and $K_{1}$ as functions of the density, one must then feed into Eqs. (39)-(41) the expression of $V_{0}$. This depends on the contact value of the radial distribution function $y_{0} \equiv g\left(r=1^{+}\right)$via Eq. (IV.2.32 b):

$$
V_{0}=6 \xi y_{0}-f^{2}+1 \text {. }
$$

For a hard-sphere gas $y_{0}$ can be determined from the equation of state via the virial equation:

$$
\frac{\beta P}{\rho}=1+4 \xi y_{0} .
$$

The requirement that both the virial and the compressibility route to the thermodynamics must give the Carnahan-Starling equation of state is then satisfied if the CarnahanStarling pressure and compressibility are substituted respectively in Eq. (143) and Eq. (10). Eqs. (42) and (41) then yield $V_{0}$ and $V_{1} / V_{0}$ as a function of density. From Eqs. (39) and (40) we finally get $z_{1}$ and $K_{1}$. 


\section{B Simulation details}

The principal aspects of the simulation and finite-size scaling techniques employed in this work have previously been detailed elsewhere in the context of a similar study of the Lennard-Jones fluid. Accordingly we confine our description to the barest essentials and refer the reader to reference [16] for a fuller account of our methods.

The Monte-Carlo simulations were performed using a Metropolis algorithm within the grand canonical ensemble [38. The MC scheme comprises only particle transfer (insertion and deletion) steps, leaving particle moves to be performed implicitly as a result of repeated transfers. To simplify identification of particle interactions a linked-list scheme was employed. This involves partitioning the periodic simulation space of volume $L^{3}$ into $m^{3}$ cubic cells, each of side the cutoff $r_{c}$. This strategy ensures that interactions emanating from particles in a given cell extend at most to particles in the 26 neighbouring cells.

In our Yukawa system the potential was cutoff at a radius $r_{c}=3.0 \sigma$, and a correction term was applied to the internal energy to compensate for the trunction. System sizes having $m=3,4,5,6$ and 7 were studied, corresponding (at coexistence) to average particle numbers of approximately 230,540,1050,1750 and 2900 respectively. For the $m=3,4$ and 5 system sizes, equilibration periods of $10^{5}$ Monte Carlo transfer attempts per cell (MCS) were utilised, while for the $m=6$ and $m=7$ system sizes up to $2 \times 10^{6}$ MCS were employed. Sampling frequencies ranged from $20 \mathrm{MCS}$ for the $m=3$ system to 150 MCS for the $m=7$ system. The total length of the production runs was also dependent upon the system size. For the $m=3$ system size, $1 \times 10^{7}$ MCS were employed, while for the $m=7$ system, runs of up to $6 \times 10^{7}$ MCS were necessary.

In the course of the simulations, the observables recorded were the particle number density $\rho=N / V$ and the energy density $u=E / V$. The joint distribution $p_{L}(\rho, u)$ was accumulated in the form of a histogram. In accordance with convention, we express $\rho$ and $u$ in reduced units: $\rho^{*}=\rho \sigma^{3}, u^{*}=u \sigma^{3}$. To allow us to explore efficiently the phase space of the model, we employed the histogram reweighting technique [39]. This method allows histogram accumulated at one set of model parameters to be reweighted to provide estimates appropriate to another set of not-too-distant model parameters.

To facilitate study of the subcritical coexistence region, the multicanonical preweighting technique [40] was employed. This technique allows one to circumvent the problems of metastability and nonergodicity that would otherwise arise from the large free energy barrier separating the coexisting phases. Details of this technique and its implementation in the fluid context are described in reference [16].

The critical point parameters were estimated using finite-size scaling technique as described in [16]. This involves matching the distribution function of the ordering operator to the independently known universal critical point form appropriate for the Ising universality class. The ordering operator is defined as $\mathcal{M} \propto\left(\rho^{*}+s u^{*}\right)$, where $s$ is a non-universal "field mixing" parameter, which is finite in the absence of particle-hole symmetry, and which is chosen to ensure that $p(\mathcal{M})$ is symmetric in $\mathcal{M}$. The estimate of the apparent critical temperature obtained by this matching procedure is, however, subject to errors associated with corrections to finite-size scaling. To deal with this, we extrapolate to the 
thermdodynamic limit using the known scaling properties of the corrections, which are expected to diminish (for sufficiently large system sizes) like $L^{-\theta / \nu}$ [16], where $\theta$ is the correction to scaling exponent and $\nu$ is the correlation length exponent. The extrapolation has been performed using a least squares fit to the data for the four largest system sizes. The results of the extrapolation are shown in figure A1, from which we estimate $T_{c}=1.212(2)$. The associated estimate for the critical density is $\rho_{c}^{*}=0.312(2)$. 
TABLE 1

\begin{tabular}{ccccc}
\hline \hline$T^{*}$ & $\rho^{*}$ & MC $^{\dagger}$ & SCOZA & LOGA/ORPA $_{\mathrm{en}}$ \\
\hline$\infty$ & 0.4 & 2.52 & 2.518 & 2.518 \\
$\infty$ & 0.6 & 4.22 & 4.283 & 4.283 \\
$\infty$ & 0.8 & 7.65 & 7.750 & 7.750 \\
2.0 & 0.4 & 1.08 & 1.120 & 1.118 \\
2.0 & 0.6 & 2.04 & 1.977 & 1.974 \\
2.0 & 0.8 & 4.27 & 4.433 & 4.432 \\
1.5 & 0.4 & 0.69 & 0.667 & 0.663 \\
1.5 & 0.6 & 1.21 & 1.220 & 1.214 \\
1.5 & 0.8 & 3.31 & 3.333 & 3.330 \\
\hline \hline
\end{tabular}

Compressibility factor $P V / N k_{B} T$ for the hard-sphere + Yukawa fluid $(z=1.8)$. Density and temperature are in reduced units $\rho^{*}=\rho \sigma^{3}, T^{*}=k_{B} T / \epsilon$, where $\sigma$ is the hard-sphere diameter and $\epsilon$ is the strength of the attractive potential. $\dagger$ : Monte Carlo data from Ref. [30]. $\diamond:$ LOGA/ORPA-energy route results from Ref. [27]. 
TABLE 2

\begin{tabular}{clrr|ll}
\hline \multicolumn{4}{c}{$\mu / k_{B} T$} \\
\hline \hline$T^{*}$ & $\rho^{*}$ & \multicolumn{1}{c}{$\mathrm{MC}^{\dagger}$} & SCOZA & \multicolumn{1}{c}{$\mathrm{MC}^{\dagger}$} & \multicolumn{1}{c}{ SCOZA } \\
\hline$\infty$ & 0.4 & $1.736(2)$ & 1.7316 & $0.1958(2)$ & 0.19744 \\
$\infty$ & 0.6 & $4.833(2)$ & 4.8147 & $0.0848(5)$ & 0.08721 \\
2.0 & 0.4 & $-0.936(2)$ & -0.9396 & $0.4992(8)$ & 0.50439 \\
2.0 & 0.6 & $0.515(2)$ & 0.5003 & $0.1594(5)$ & 0.15976 \\
1.5 & 0.4 & $-1.823(2)$ & -1.8258 & $0.968(3)$ & 1.0150 \\
1.5 & 0.6 & $-0.905(2)$ & -0.9294 & $0.2217(5)$ & 0.22147 \\
\hline \hline
\end{tabular}

Chemical potential $\mu$ and reduced compressibility $\chi_{\text {red }}$ of the hard-sphere Yukawa fluid $(z=1.8)$. Density and temperature are in reduced units. $\dagger$ : Monte Carlo simulation performed in this work. The numbers in brackets give the error in the last figure. 
TABLE 3

\begin{tabular}{cccccc}
\hline \hline$T^{*}$ & $\rho^{*}$ & $\mathrm{MC}^{\dagger}$ & $\mathrm{MC}^{\ddagger}$ & SCOZA & LOGA/ORPA $^{\diamond}$ \\
\hline$\infty$ & 0.4 & -2.495 & $-2.516(2)$ & -2.517 & -2.517 \\
$\infty$ & 0.6 & -3.975 & $-4.002(2)$ & -4.002 & -4.002 \\
$\infty$ & 0.8 & -5.573 & & -5.611 & -5.611 \\
2.0 & 0.4 & -2.583 & $-2.595(2)$ & -2.583 & -2.574 \\
2.0 & 0.6 & -4.030 & $-4.036(2)$ & -4.030 & -4.026 \\
2.0 & 0.8 & -5.622 & & -5.620 & -5.618 \\
1.5 & 0.4 & -2.622 & $-2.640(2)$ & -2.623 & -2.602 \\
1.5 & 0.6 & -4.051 & $-4.053(2)$ & -4.043 & -4.036 \\
1.5 & 0.8 & -5.630 & & -5.623 & -5.621 \\
1.0 & 0.6 & -4.073 & & -4.097 & -4.065 \\
1.0 & 0.8 & -5.635 & & -5.631 & -5.628 \\
\hline \hline
\end{tabular}

Internal energy per particle of the hard-sphere Yukawa fluid. All quantities are in reduced units. $\dagger$ : Monte Carlo simulation of Ref. [30]. ‡: Monte Carlo simulation performed in this work. The number in brackets give the error in the last figure. $\diamond:$ LOGA/ORPA. The entries for $T^{*}=\infty$ are from this work, the rest are from Ref. [26]. 
TABLE 4

\begin{tabular}{cccccccc}
\hline \hline & $\mathrm{MC}^{\circ}$ & $\mathrm{MC}^{\diamond}$ & $\mathrm{MC}^{\dagger}$ & SCOZA $_{1-\text { Yuk }}^{\ddagger}$ & SCOZA $^{\prime}$ & HMSA $^{\bullet}$ & MHNC $^{\bullet}$ \\
\hline$\rho_{c}^{*}$ & 0.294 & 0.313 & $0.312(2)$ & 0.308 & 0.314 & 0.36 & 0.28 \\
$T_{c}^{*}$ & 1.192 & 1.178 & $1.212(2)$ & 1.201 & 1.219 & 1.25 & 1.21 \\
\hline \hline
\end{tabular}

Critical density and temperature (in reduced units) for the hard-sphere Yukawa fluid. $\circ$ : MC simulation of Ref. [31]. $\diamond$ : MC simulation of Ref. [32] $\dagger$ : MC simulation performed in this work. $\ddagger$ : SCOZA with 1-Yukawa $c(r)$ (see text). •: from Ref. 6] 


\section{FIGURE CAPTIONS}

Fig. 1 Compressibility factor $Z=P /\left(\rho k_{B} T\right)$ of the hard-sphere Yukawa fluid $(z=1.8)$ as a function of the reduced density $\rho^{*}$ along two isotherms at reduced temperature $T^{*}=2$ (upper curve) and $T^{*}=1.5$ (lower curve). Full curve: SCOZA. Squares: $\mathrm{MC}$ simulation results [30].

Fig. 2 Coexistence curve of the hard-sphere Yukawa fluid $(z=1.8)$ in the densitytemperature plane. Density and temperature are in reduced units. Full curve: SCOZA. Dashed curve: SCOZA with a one-Yukawa direct correlation function $c(r)$ (see text). Squares: MC results (this work).

Fig. 3 Coexistence curve of the hard-sphere Yukawa fluid in the internal energy-temperature plane. $E^{*} / N$ is the internal energy per particle in reduced units. Notation as in Fig. 2.

Fig. 4 Coexistence curve of the hard-sphere Yukawa fluid in the temperature-chemical potential plane. All quantities are in reduced units. Notation as in Fig. 2.

Fig. 5 Log-log plot of the reduced compressibility $\chi_{\text {red }}$ of the hard-sphere Yukawa fluid $(z=1.8)$ on the critical isochore as a function of the reduced temperature $t=$ $\left(T-T_{c}\right) / T_{c}$ according to the SCOZA (a) and effective exponent $\gamma_{\text {eff }}$, defined as $\gamma_{\text {eff }}=-d\left(\log \chi_{\mathrm{red}}\right) / d(\log t)(\mathrm{b})$.

Fig. A1 The apparent critical temperature, (as defined by the matching condition described in the text), plotted as a function of $L^{-(\theta+1) / \nu}$, with $\theta=0.54$ and $\nu=0.629$. The extrapolation of the least squares fit to infinite volume yields the estimate $T_{c}^{*}=1.212(2)$. 


\section{References}

[1] Barker, J. A., and Henderson, D., 1967, J. Chem. Phys. 47, 2856, 4714. These are parts I and II of a study entitled Perturbation Theory and Equation of State for Fluids, with subtitles The Square-Well Potential and A Successful Theory of Fluids respectively.

[2] See, e.g., Mansoori, G. A., and Canfield, F. B., 1969, J. Chem. Phys. 51, 4958; Rasaiah, J., and Stell, G., 1970, Mol. Phys. 18, 249; Weeks, J. D., Chandler, D., and Andersen, H. C., 1971, J. Chem. Phys. 54, 5237; 55, 5421.

[3] Hauge, E. H., and Hemmer, P. C., 1966, J. Chem. Phys. 45, 223.

[4] Caccamo, C., 1996, Phys. Reports 274, 1, provides an excellent description of modern integral-equation theories of $g(r)$.

[5] Rosenfeld, Y., and Ashcroft, N. W., 1979, Phys. Rev A 20, 1208.

[6] Caccamo, C., Giunta, G., and Malescio, G., 1995, Mol. Phys. 84, 125.

[7] Caccamo, C., 1995, Phys. Rev. B 51, 3387.

[8] Caccamo, C., Giaquinta, P. V., and Giunta, G., 1993, J. Phys.: Condens. Matter 5, B75

[9] Zerah, G., and Hansen, J. P., 1985, J. Chem. Phys. 84, 2336.

[10] Høye, J. S., Pini, D., and Stell, G., unpublished.

[11] Dickman, R., and Stell, G., 1996, Phys. Rev. Lett. 77, 996.

[12] Pini, D., Stell, G., and Dickman, R., 1998, Phys. Rev. E, in press.

[13] Høye, J. S., and Stell, G., 1977, J. Chem. Phys. 67, 439.

[14] Høye, J. S., and Stell, G., 1984, Mol. Phys. 52, 1071.

[15] Pini, D., Stell, G., and Høye, J. S., submitted to Int. J. Thermophys.

[16] Wilding, N. B., 1995, Phys. Rev. E 52, 602; Wilding, N.B. Bruce, A.D., 1992, J. Phys. Condens. Matter 4, 3087.

[17] Stell, G., 1971, J. Chem. Phys. 55, 1485.

[18] Andersen, H. C., and Chandler, D., 1972, J. Chem. Phys 57, 1918.

[19] Verlet, L., and Weis, J. J., 1972, Phys. Rev. A 5, 939; Henderson, D., and Grundke, E. W., 1975, J. Chem. Phys. 63, 601.

[20] Waisman, E., 1973, Mol. Phys. 25, 45. 
[21] Høye, J. S., and Stell, G., 1976, Mol. Phys. 32, 195.

[22] Henderson, D., Stell, G., and Waisman, E., 1975, J. Chem. Phys. 62, 4247; Henderson, D., and Blum, L., 1976, Mol. Phys. 32, 1627.

[23] Høye, J. S., Stell, G., and Waisman, E., 1976, Mol. Phys. 32, 209.

[24] Høye, J. S., and Stell, G., 1984, Mol. Phys. 52, 1057.

[25] Konior, J., and Jedrzejek, C., 1983, Mol. Phys. 48, 219.

[26] Konior, J., and Jedrzejek, C., 1985, Mol. Phys. 55, 187.

[27] Konior, J., 1989, Mol. Phys. 68, 129.

[28] Ames, W. F., 1977, Numerical Methods for Partial Differential Equations (New York: Academic Press).

[29] Stell, G., and Penrose, O., 1983, Phys. Rev. Lett. 51, 1397.

[30] Henderson, D., Waisman, E., Lebowitz, J. L., Blum, L., 1978, Mol. Phys. 35, 241.

[31] Smit, B., and Frenkel, D., 1991, Mol. Phys. 74, 35.

[32] Lomba, E., and Almarza, N. G., 1994, J. Chem. Phys. 100, 8367.

[33] Borge, A., and Høye, J. S., 1998, J. Chem. Phys., in press.

[34] Tau, M., and Reatto, L., 1985, J. Chem. Phys. 83, 1921.

[35] Høye, J. S., and Stell, G., 1997, Physica A, 244, 176; 247, 497.

[36] Kierlik, E., Rosinberg, M. L., and Tarjus, G., 1997, J. Stat. Phys. 89, 215.

[37] Kierlik, E., Rosinberg, M. L., and Tarjus, G., preprint

[38] Adams, D. J., 1975, Mol. Phys. 29, 307.

[39] Ferrenberg, A. M., and Swendsen, R. H., 1988, Phys. Rev. Lett. 61, 2635; ibid 1989, 63, 1195.

[40] Berg, B., and Neuhaus, T., 1992, Phys. Rev. Lett. 68, 9. 


$$
\mathrm{Z}=\mathrm{P} /\left(\rho \mathrm{k}_{\mathrm{B}} \mathrm{T}\right)
$$






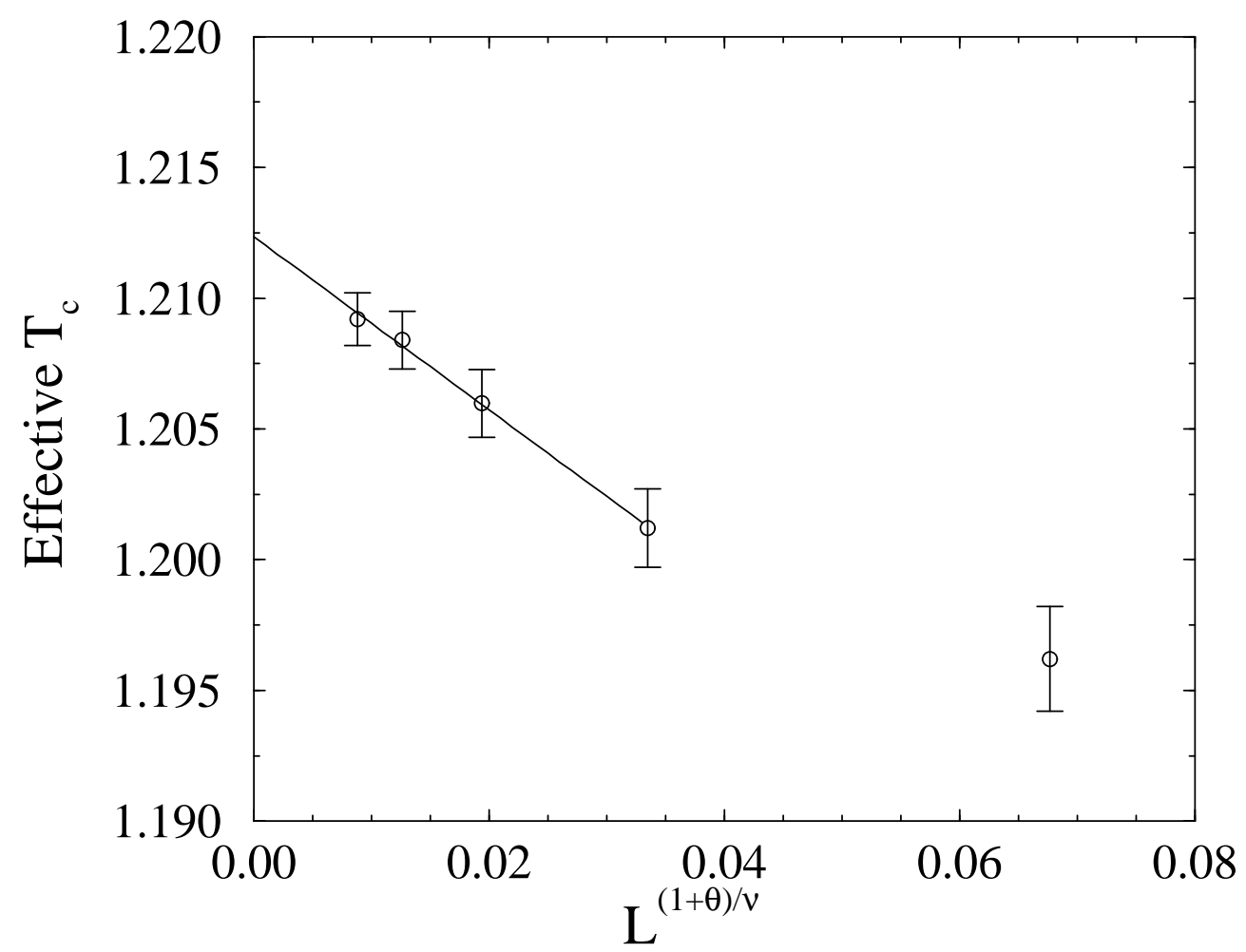




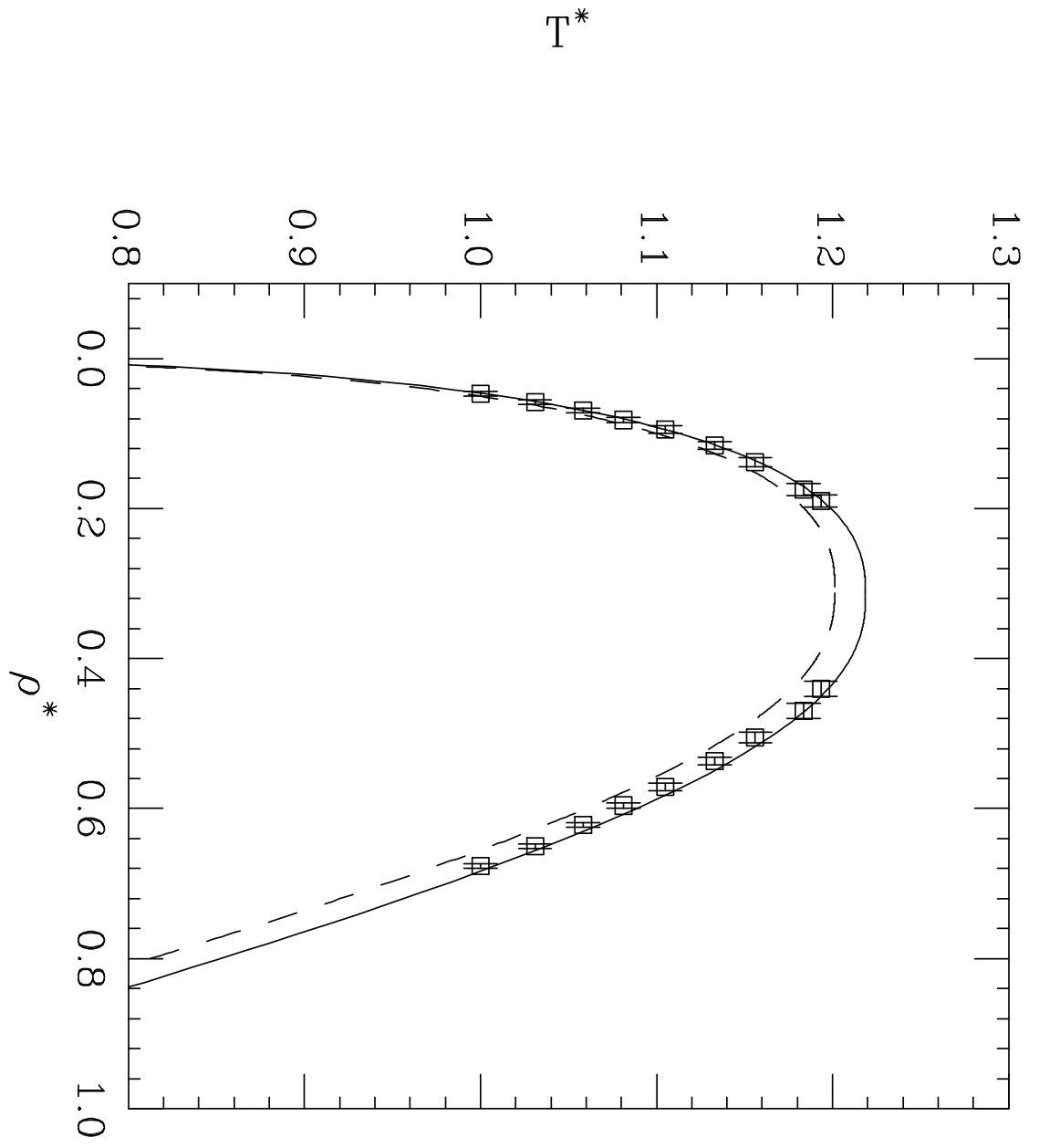




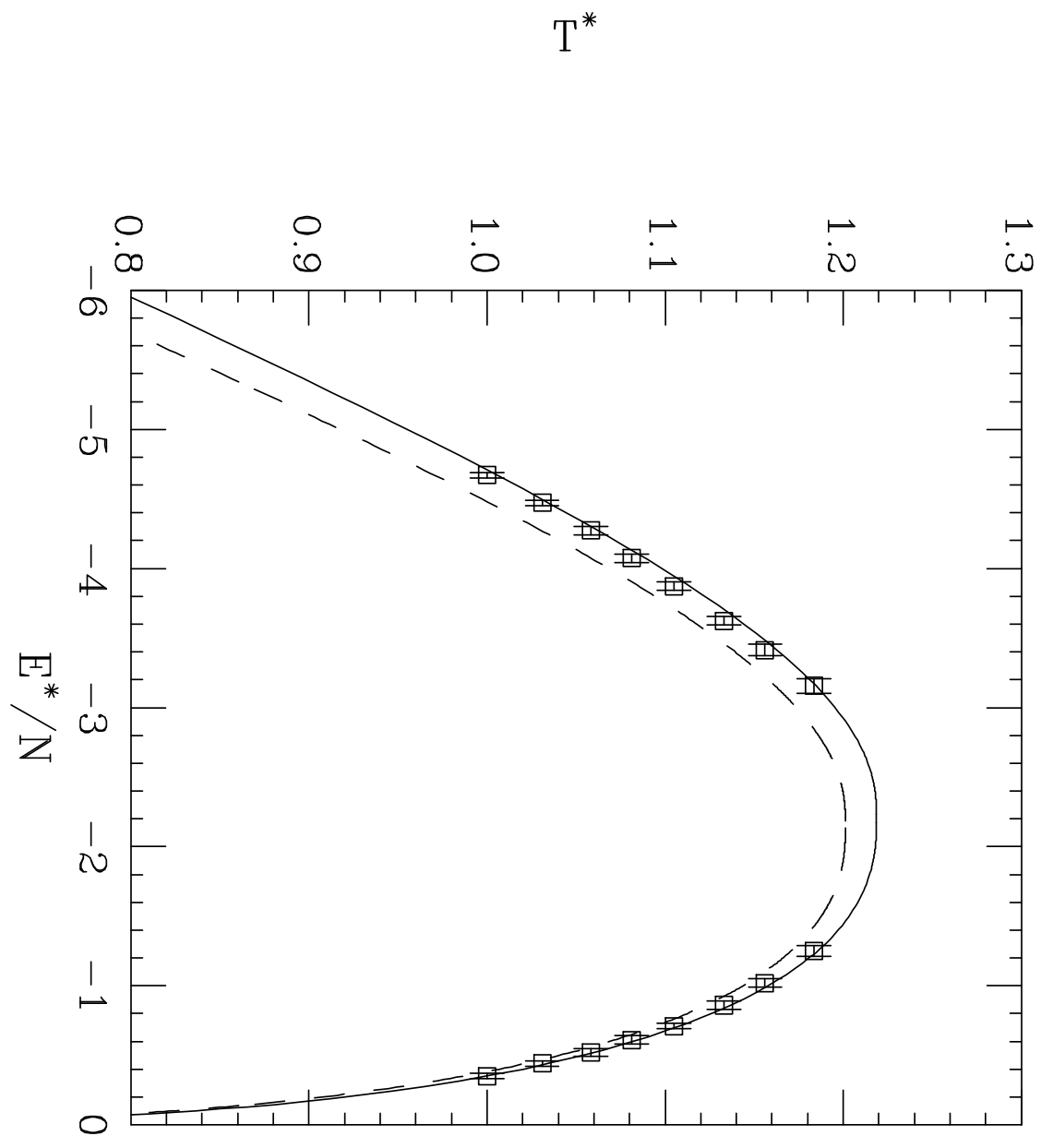




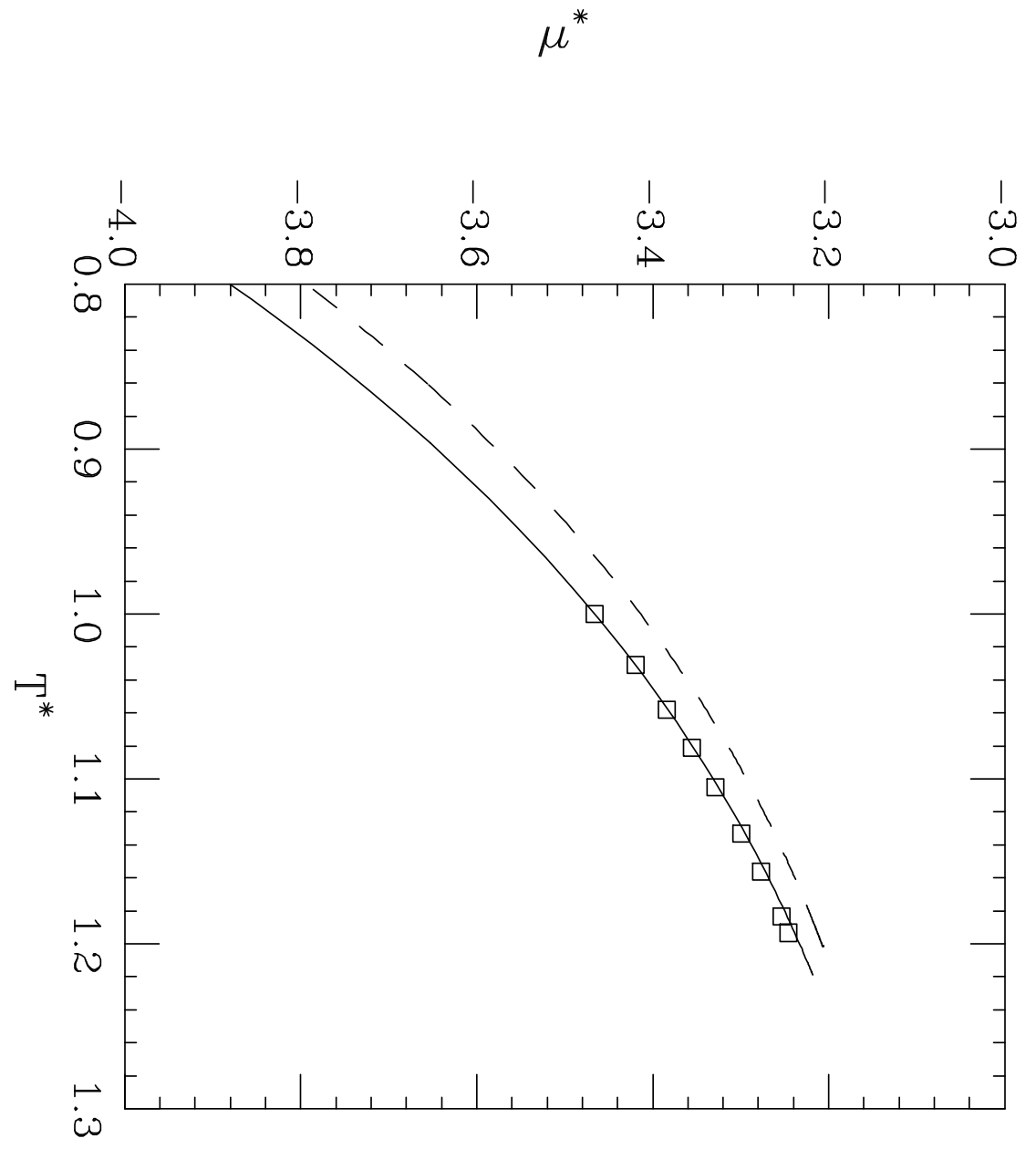


$(7)^{01801}$

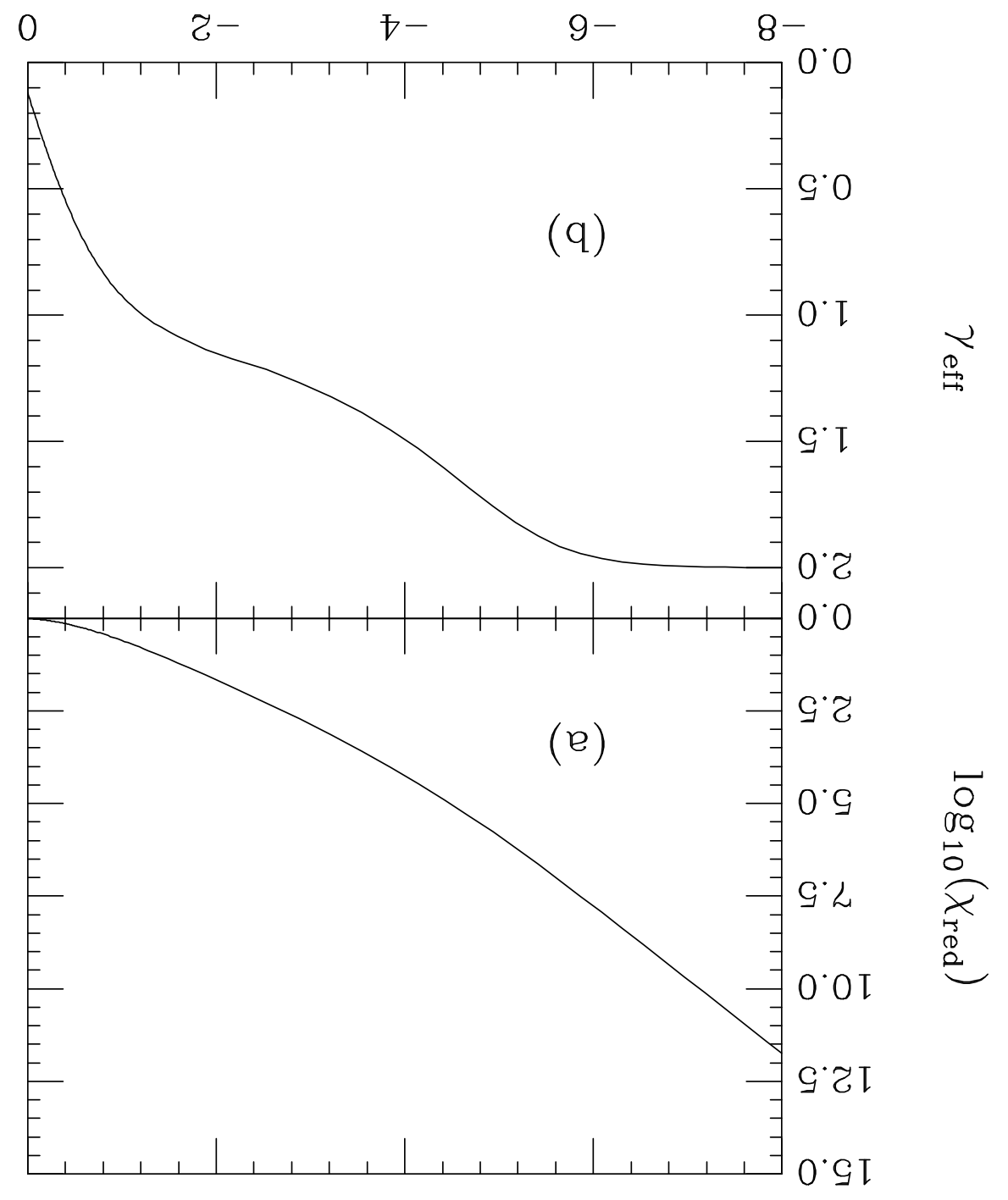

